\title{
Avances y desafíos de la gestión documental en las organizaciones
}

Beatriz Mercado', Bibliotecaria Documentalista, Universidad Tecnológica Metropolitana

Coordinadora Académica, carrera de Gestión de Información, Bibliotecología y Archivística (GIBA) y Diplomado de Archivística

La gestión documental es un aspecto clave para la eficiencia y productividad de las organizaciones. Este artículo aborda la importancia y beneficios asociados a la implementación de sistemas de gestión documental. También se revisan las principales problemáticas y obstáculos que se presentan en este tipo de iniciativas y cómo, a nivel internacional, han surgido una serie de directrices en forma de normas de gestión para apoyar a las organizaciones bajo una metodología de mejora continua. Se hace referencia, en particular, a la más reciente, la serie ISO 30300, que además permite que la organización acredite ante sus pares la adopción de las mejores prácticas en el ámbito documental.

\section{INTRODUCCIÓN}

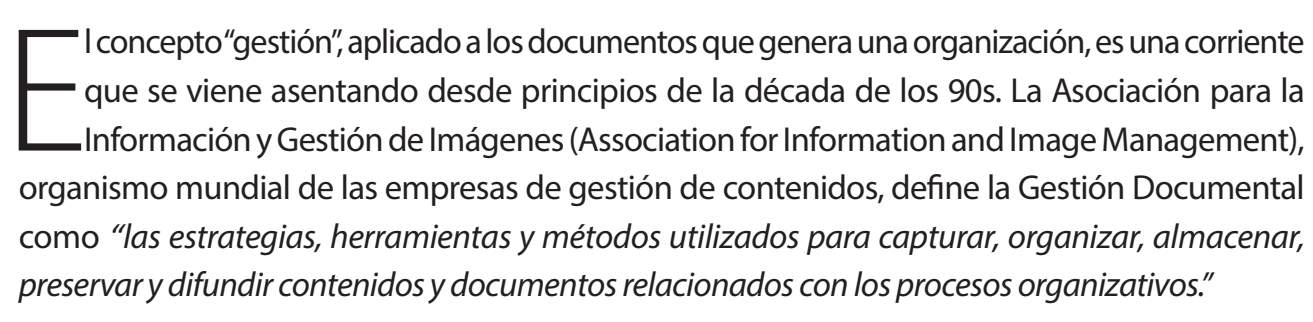

A pesar de que el hecho de confundir gestión documental con algún tipo de sistema informático está bastante arraigado en los círculos menos especializados, la gestión documental es mucho más que las soluciones tecnológicas que soportan sus procesos. En efecto, su esencia está en el establecimiento de políticas, prácticas, procedimientos y responsabilidades que, desde lo estratégico a lo operativo, permiten que una organización coordine

(1) Email de contacto: bmercado@uahurtado.cl 
y controle todas aquellas funciones $y$ actividades específicas que afectan la creación, recepción, ubicación, acceso y preservación de los documentos. Todas estas acciones, además, deben considerar la protección de las características estructurales y contextuales de los documentos, a fin de garantizar su autenticidad, fiabilidad, integridad y disponibilidad a lo largo del tiempo.

El presente artículo se enfoca precisamente a cómo la comunidad internacional ha avanzado en la definición y sistematización de directrices que, independiente del rubro o tipo de institución, apoyan la definición de sistemas que permiten que la organización utilice y reutilice su capital documental. La amplitud y transversalidad de procesos de gestión documental en una organización justifica que ésta invierta en la implementación de las mejores prácticas que, desde el año 2001, es posible encontrar sistematizadas en una serie de normas de aplicación voluntaria. Más aún, a partir del año 2011, las organizaciones pueden acreditar ante sus pares el uso de estas prácticas implementando la serie de normas ISO 30300.

\section{Importancia y problemáticas de la gestión documental en las organizaciones}

Toda organización, en su actividad diaria, genera y recibe documentos que constituyen evidencias de dicha actividad. Además de este valor probatorio, los documentos tienen valor por la información que contienen. Esta información es susceptible de generar conocimiento: el know how de la empresa. Ambos valores emergen y pueden ser explotados siempre y cuando la organización disponga de un buen sistema de gestión documental.

En particular, el valor probatorio está directamente asociado a "evidenciar" el cumplimiento de los procedimientos establecidos por la organización para el desarrollo de sus actividades, las que además de la propia normativa interna, deben respetar el marco reglamentario que aplica tanto a su rubro específico, como a procesos de apoyo trasversales que es posible identificar en toda organización (recursos humanos, finanzas, tecnología, entre otros). Este marco reglamentario incluye las leyes, la jurisprudencia, y la reglamentación que regula tanto el marco organizativo en general, como el que afecta a cada sector en particular. Incluso, es posible extender este cumplimiento a normas y buenas prácticas de aplicación voluntaria (de calidad, por ejemplo) o a expectativas de la sociedad acerca de lo que se considera como un comportamiento aceptable en relación con el sector específico u organización. Así, son muchos los documentos que pueden actuar como prueba en caso de conflicto; los ejemplos son innumerables: facturas, proyectos arquitectónicos, pólizas de seguros, contratos, matrículas de estudiantes, expedientes académicos, expedientes judiciales, permisos de obra, escrituras de propiedad, entre muchísimos otros. (García Alsina, 2011).

Las necesidades legales, las responsabilidades contractuales y el aumento de las capacidades de almacenamiento impulsan la acumulación de documentos en organizaciones de todo tipo. Lo anterior redunda en que, según un estudio publicado en la Revista de AENOR2, empleamos más de 50 minutos al día en buscar documentos e información. Y es que, la gestión de información o documentos es parte integral de los procesos, servicios y sistemas de gestión de cualquier entidad; incluso para muchas organizaciones está indisolublemente asociada al propio negocio. (Valderrama, 2016).

Esto último se hace más tangible cuando analizamos los más importantes sistemas tecnológicos empresariales, como por ejemplo los de tipo ERP3. Estas herramientas no sólo son capaces de gestionar la documentación administrativa propiamente tal, sino que además pueden controlar los flujos de trabajo en la tramitación de documentos y expedientes, capturar información desde bases de datos de producción, contabilidad y otros para la generación de nuevos documentos, y posibilitar búsquedas por muchos criterios a fin de ubicar oportunamente la información que la organización necesita. La integración de los documentos con las transacciones permite una visión clara del proceso de negocio (independientemente de su fuente y formato), y de las personas que actúan en dicho proceso (dentro y fuera de la organización), asegurando el trabajo sobre un contenido único.

\section{La realidad chilena}

Lamentablemente, la realidad en Chile dista bastante respecto de este último escenario. En nuestro país, son pocas las empresas que han alcanzado la integración de sus procesos de negocio con sus procesos de gestión de documentos. Por el contrario, lo común es encontrar que la información

(2) Asociación Española de Normalización y Certificación.

(3) Un ERP (Enterprise Resource Planning) es un sistema integrado de gestión y de datos único, donde converge toda la información de una empresa y ésta es utilizada para tomar decisiones, especialmente las que tienen que ver con la producción, la logística, el inventario, los envíos y la contabilidad. 
está aislada. La documentación en papel, los correos electrónicos, los archivos almacenados en carpetas en los computadores personales. Los datos gestionados por sistemas ERP no están relacionados entre sí, la información no se comparte y la pérdida y falta de control de información generan ineficacia y descontrol en las organizaciones. (San Servando, 2013).

Sin embargo, son cada vez más las organizaciones que entienden el riesgo que enfrentan al no poder localizar y acceder a los documentos que necesitan y en el momento que los necesitan. Esta realidad justifica el diseño e implementación de un sistema de gestión documental eficiente, que permita crear, registrar, clasificar, facilitar y controlar el acceso, almacenar, seguir el trazo y conservar o "saber eliminar" documentos. (García Alsina, 2011).

Aunque en una lectura rápida, los aspectos antes mencionados pudieran parecer simples, la verdad es que son bastante complejos, sobre todo al estimar que cada persona en una organización genera y/o recibe al menos un documento por cada tarea unitaria que realiza. La complejidad aumenta cuando consideramos que estos documentos pueden ser generados en múltiples soportes y formatos que deben convivir, relacionarse y, en algunos casos, interoperar entre sí, tanto dentro de la misma organización como con aquellas personas e entidades externas con las que interactúan (clientes, proveedores, entes reguladores, por mencionar algunos).

Resulta evidente que en este complicado escenario es altamente deseable para las organizaciones contar con un consolidado de buenas prácticas en el manejo de sus documentos, que incluya todos los procesos y controles documentales, desde la creación del documento o incluso antes. Tampoco es de extrañar, por la universalidad de la problemática, que la iniciativa naciera de una de las organizaciones mejor posicionada a nivel internacional en normalización: la ISO.

\section{Normalización y certificación de los sistemas de gestión en organizaciones: la experiencia de la ISO}

La Organización Internacional de Normalización, más conocida por su acrónimo en inglés ISO, es la organización internacional más prestigiosa en normalización. Su fuerza se basa en estrictos procedimientos de aprobación de normas y el reconocimiento internacional de las mismas. Es una organización nogubernamental establecida en 1947 y su misión es promover el desarrollo de la estandarización y actividades relacionadas, con el propósito de facilitar el intercambio internacional de bienes y servicios, y para desarrollar la cooperación en la esfera de la actividad intelectual, científica, tecnológica y económica. ${ }^{4}$ Con una Secretaría Central que tiene su sede en Ginebra, Suiza, la componen 162 países a través de sus respectivos organismos nacionales de normalización. En Chile, dicha responsabilidad recae en el Instituto Nacional de Normalización, INN, formando parte de la ISO de manera ininterrumpida desde su fundación.

En términos generales, la normalización es una actividad colectiva encaminada a establecer soluciones a situaciones repetitivas $y$, por ende, se traduce en la elaboración, difusión y aplicación de normas que, en su mayoría, son de aplicación voluntaria.

La normalización, tal y como la conocemos hoy, nace en el siglo XX como respuesta a los avances de la industria para establecer un conjunto de disposiciones estandarizadas que faciliten la producción. Por lo mismo, desde sus inicios, las normas ISO (como muchas otras) se centraron en la normalización de productos, cubriendo prácticamente todos los sectores industriales, a fin de unificar criterios, dimensiones y propiedades que debían cumplir estos productos. Sin embargo, a partir de la década de los 90s, la ISO se inició en el camino de la normalización de la gestión de las organizaciones. Las normas de gestión, más que enfocarse al producto final y sus procesos productivos, centran su atención en la mejora continua del desempeño global de la organización como un objetivo permanente $y$, en la metodología que promueven, son esenciales aspectos como el enfoque al cliente, el liderazgo, la participación del personal, el enfoque de procesos, el enfoque de sistemas, el enfoque basado en hechos.

El impacto y reconocimiento de las normas de gestión fue casi inmediato. El mejor ejemplo es el éxito que a nivel internacional adquirió la serie de normas de calidad ISO 9000, por la cual la ISO es comúnmente reconocida fuera de los círculos especializados. Lo anterior, dio pie a la elaboración de normas de gestión en otros ámbitos más específicos como la serie ISO 14000 (sistema de gestión medioambiental), la serie ISO 27000 (sistema de gestión de seguridad de la información) y la serie ISO 31000 (sistema de gestión de riesgos).

Otra característica importante es que los sistemas de gestión son normas certificables, es decir, que están redactadas en forma de requisitos de manera que una entidad certificadora pueda verificar y acreditar

(4) www.iso.org 
su cumplimiento. Estas entidades certificadoras que, a su vez, han sido validadas por el órgano normalizador (ISO), son empresas que actúan en el mercado compitiendo libremente por captar clientes. Estos clientes pagan por el proceso de certificarse, lo que supuestamente les ayuda en su reputación, en cumplir determinados requisitos para la contratación o en aumentar sus ventas como beneficios añadidos de la propia implantación del sistema de gestión (Bustelo, 2012).

No es de extrañar, entonces, que un pilar fundamental en la gestión de una organización como son los documentos que registran sus actividades, haya sido punto de mira para el desarrollo de una norma de gestión. Así nació el proyecto ISO 30300, que propone una metodología normalizada para la gestión de las organizaciones desde el punto de vista de los documentos.

\section{La serie ISO 30300}

Con la misma estructura y sistema de codificación de las demás normas ISO de gestión, desde finales del año 2011 se han publicados las tres primeras normas que pasarían a conformar la serie ISO 30300 (sistema de gestión para los documentos):

ISO 30300: Especifica la terminología para toda la serie de normas, los objetivos y los beneficios de un sistema de gestión para los documentos. Esta es una norma introductoria de toda la serie. Define el vocabulario o terminología que se va a utilizar en el resto de las normas y presenta la justificación y enfoque de toda la serie.

ISO 30301: Especifica los requisitos para implementar un sistema de gestión para los documentos (norma certificable). En esta norma se establecen los requisitos para la implementación de un sistema de gestión de documentos y sobre los que se realizarán las evaluaciones y posibles certificaciones. Todas las demás normas de la serie se refieren a esta norma principal.

ISO 30302: Esta norma, incorporada en octubre de 2015, proporciona una guía de implementación concordante con los requisitos establecidos en la norma ISO 30301 y que, sin duda, es de una ayuda inestimable para toda organización que tenga por objetivo certificarse.

Una ventaja apreciable de la serie ISO 30300 es la complementariedad y compatibilidad con el resto de normas de sistemas de gestión (ISO 9000, ISO 14000, ISO 27000, etc.) al determinar, en su modelo básico, los requisitos de gestión documental y las expectativas de las partes interesadas (ver fig. 1). Este enfoque integrador facilita y reduce los esfuerzos de implementación, cuando la organización ya ha aplicado (o certificado) otro sistema de gestión ISO (Bustelo, 2011).

\section{Desafíos pendientes de la gestión documental en las organizaciones chilenas}

Llegados a este punto, se hace fundamental analizar cómo esta norma enfoca las principales problemáticas que enfrentan las organizaciones en nuestro país en el manejo de sus documentos. Este enfoque de gestión probablemente afecte los cimientos de la cultura de la mayoría de las organizaciones, que tienden a considerar los documentos sólo como el residuo de su labor administrativa y no le asignan la importancia que tienen.

Además de varios requisitos asociados a la planificación, soporte, operación, control y evaluación del desempeño del sistema de gestión de documentos, la norma releva dos aspectos sobre los cuales quiero referirme, porque son los

\section{Figura 1}

Estructura del Sistema de Gestión para los documentos

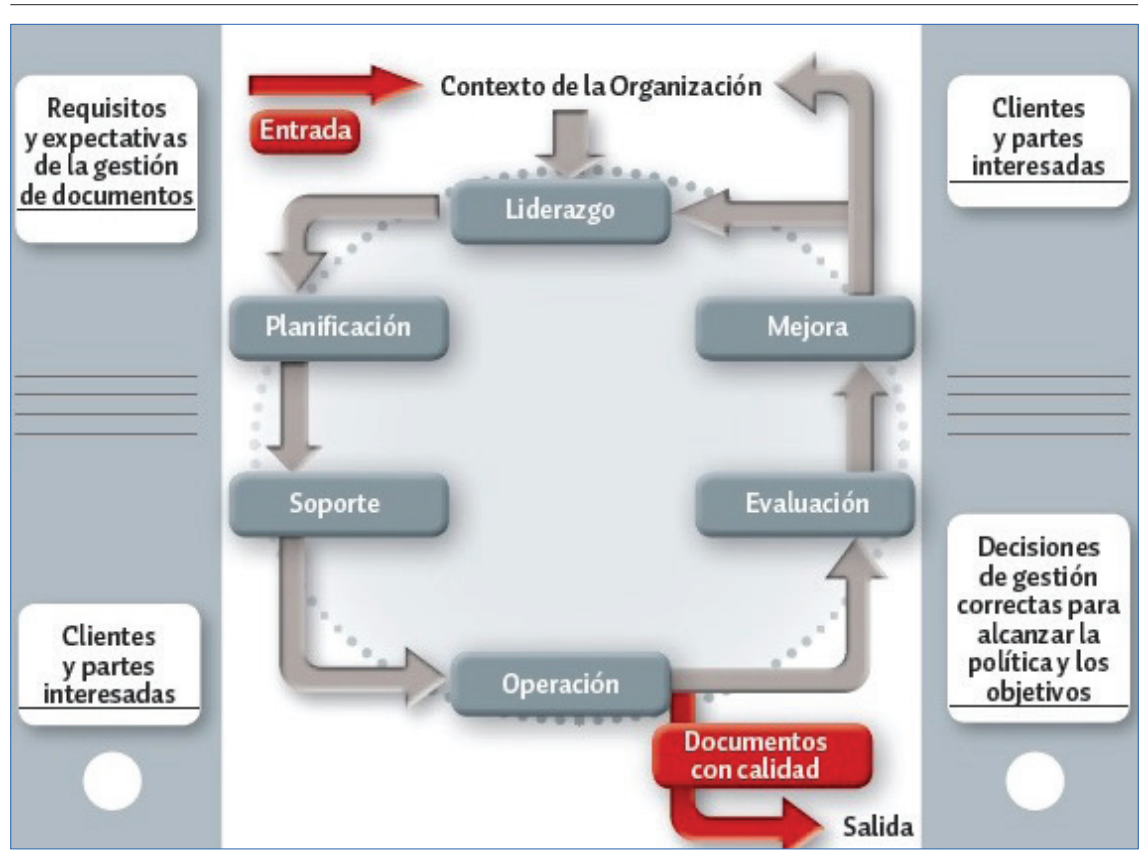

FUENTE: AENOR. (2011). UNE-ISO 30301. Información y Documentación - Sistemas de Gestión para los Documentos - Requisitos. 
que habitualmente se pasan por alto o no se les da la suficiente importancia al momento de diseñar e implementar el sistema: el contexto de la organización y el liderazgo.

Respecto del contexto de la organización, la norma pone de manifiesto que no se puede concebir a la gestión de documentos como un ente aislado del entorno externo e interno de la organización, ya que éstos tienen una incidencia fundamental respecto de las decisiones que se tomen, especialmente en lo que tiene que ver con la conservación y disposición documental. Es decir, ¿cómo y por cuánto tiempo mantendremos los documentos en la organización? Lo que habitualmente es posible observar, tanto en organizaciones públicas como privadas, es que no hay ningún tipo de selección sobre lo que debe conservarse y, menos aún, que los criterios de selección hayan sido documentados e institucionalizados. Así, la gestión que puede hacerse sobre espacios de almacenamiento (normalmente bodegas y depósitos que no cumplen los mínimos estándares para garantizar una adecuada conservación de los documentos) es prácticamente nula. La actitud es aún más despreocupada en el caso de los documentos electrónicos, por la falsa creencia que los espacios de almacenamiento son inagotables.

Los documentos evidencian las operaciones. Son el testimonio de que una organización cumple la normativa que la regula y, por ende, un adecuado sistema de gestión de documentos debe evaluar los requisitos de negocio, legales y reglamentarios que debe cumplir en sus operaciones, a fin de conservar los documentos sólo durante el tiempo que los precise. Incluso, los requisitos avanzan un paso más, al delegar al sistema de gestión de documentos la responsabilidad de detectar cuando alguna actividad no está siendo adecuadamente documentada y revierta dicha situación.

Además de esta función probatoria frente a su contexto externo, los documentos también son evidencia de las capacidades internas de la organización para dar cumplimento a sus políticas, objetivos, estrategias, procedimientos y modelos adoptados. La toma de decisiones informada y la mejora continua de una organización son consecuencia de una adecuada documentación y registro de los procesos.

Cumplir requisitos asociados a lo antes expuesto implica un cambio radical, ya que eleva la gestión documental a un nivel que raras veces se puede apreciar en las organizaciones en nuestro país, al plano estratégico, sacándola del habitual rol de apoyo o soporte. Esto nos lleva al segundo aspecto que vale la pena destacar: el de liderazgo y el compromiso que debe adoptar la más alta dirección de la organización en los procesos documentales.

Las orientaciones que emanen de la alta dirección, afianzando la importancia de los procesos documentales, son imprescindibles. Estas orientaciones deben llegar a todos los "rincones" de la organización que están permanentemente creando documentos que registran sus actividades. En casi la totalidad de los casos, los documentos son gestionados por personas que fueron contratadas para realizar otras funciones que no consideraban acciones asociadas a la gestión documental (más raro aún sería encontrarlas en sus perfiles de cargo), por lo que la mal entendida sobrecarga de trabajo debe estar adecuadamente justificada y encausada desde el nivel gerencial.

Pero este compromiso desde la alta dirección no sólo puede ser una declaración, aunque sea documentada a través de una política, debidamente comunicada a todos los niveles como exige la norma. Para que sea sentido e incorporado, debe palparse con hechos, como la creación de un rol específico con formación y competencias a fin de que actúe como representante de la gestión documental en la organización. El compromiso también debe estar respaldado a través de la asignación de presupuestos que permitan el desarrollo permanente de proyectos orientados a la mejora de condiciones, tecnológicas y de infraestructura principalmente. Por último, también debe lograr el involucramiento real de los mandos medios que, muchas veces enfocados en el desempeño de sus propias áreas, tienden a ser los principales enemigos de la consolidación de los procesos de gestión documental, al relegarlos frente a metas locales.

\section{Conclusiones}

Concebir los procesos documentales como un sistema que involucra a toda la organización y no como actividades aisladas a cargo de unos pocos, es uno de los factores de éxito de la implementación de un sistema de gestión documental. Lo anterior está ampliamente avalado por la norma ISO 30300 y, por ende, reconocido internacionalmente.

Una organización que esté dispuesta a implementar esta norma e, idealmente, certificarla, requiere del convencimiento al más alto nivel de que los beneficios que obtendrá superarán ampliamente a los costos de implementación. Estos beneficios tarde o temprano redundarán en lo económico: al optimizar procesos; al no estar expuesto a conflictos sin los debidos respaldos; al aumentar la ventaja competitiva, entre otros. 
Aun así, la implementación no será sencilla, ni rápida, ya que involucra a la propia cultura organizacional, que puede estar o no dispuesta al cambio que significa considerar que los documentos ya no son papeles viejos o documentos electrónicos desperdigados en los diferentes computadores personales, respecto de los cuales no vale la pena perder el tiempo. Es fundamental, instalar el conocimiento de que los documentos son un activo más de la organización, donde todos y cada uno de sus miembros tienen responsabilidades en su gestión.

\section{Referencias}

AENOR. (2006). UNE-ISO 15489-1. Información y Documentación Gestión de Documentos - Parte I: Generalidades. Madrid: AENOR.

AENOR.(2011).UNE-ISO30301.Información y Documentación - Sistemas de Gestión para los Documentos Requisitos. Madrid: AENOR.

Bustelo Ruesta, Carlota. (2012). La normalización internacional en información y documentación: ¿una historia de éxitos? El caso de la normalización ISO en gestión de documentos. En: Métodos de información (MEI), II Época, Vol. 3, no, 4, 2012, pp. 39-46. Disponible en: http://dx.doi.org/10.5557/ IIMEI2-N2-039046

Bustelo Ruesta, Carlota. (2011). Serie ISO 30300: Sistema de Gestión para los Documentos.Madrid:SEDIC,Asociación Española de Documentación e Información Científica. Documentos de Trabajo. $36 \mathrm{p}$.

García Alsina, Monserrat. (2011). El valor oculto de los documentos: razones de su gestión. Revista de los Estudios de Ciencias de la Información y de la Comunicación, 4. Disponible en: http://www.uoc.edu/divulgacio/ comein/es/numero04/articles/ Article-Montserrat-Garcia-Alsina.html Gauchi Risso, Verónica (2012). Aproximación teórica a la relación entre los términos gestión documental, gestión de información y gestión del conocimiento. Revista Española de Documentación Científica, (35) 4: 531-554. Disponible en: http:// redc.revistas.csic.es/index.php/ redc/article/viewFile/762/844

San Servando Hernández, Nuria (2013). Gestión documental para Pymes. La Coruña, NETBIBLIO, 115 p. Disponible en: http://www. fundacioninnovacionrioja.com/ uploads/media/GD_WEB.pdf

Valderrama, José Ángel. (2012). Sistemas de gestión: objetivos y estructura. En: Jornada Clave de las ISO 30300 para profesionales de la Gestión de Documentos. Madrid, 21 de mayo de 2012. Disponible en: http://www. mecd.gob.es/cultura-mecd/en/ $\mathrm{dms} / \mathrm{mecd} /$ cultura-mecd/areascultura/principal/novedades/ archivos/2012/normas-iso-30300/ Jornada30300_valderrama.pdf

Valderrama, José Ángel. (2016). La gestión de documentos como estrategia empresarial. Revista AENOR, mayo de 2016: 26-29.

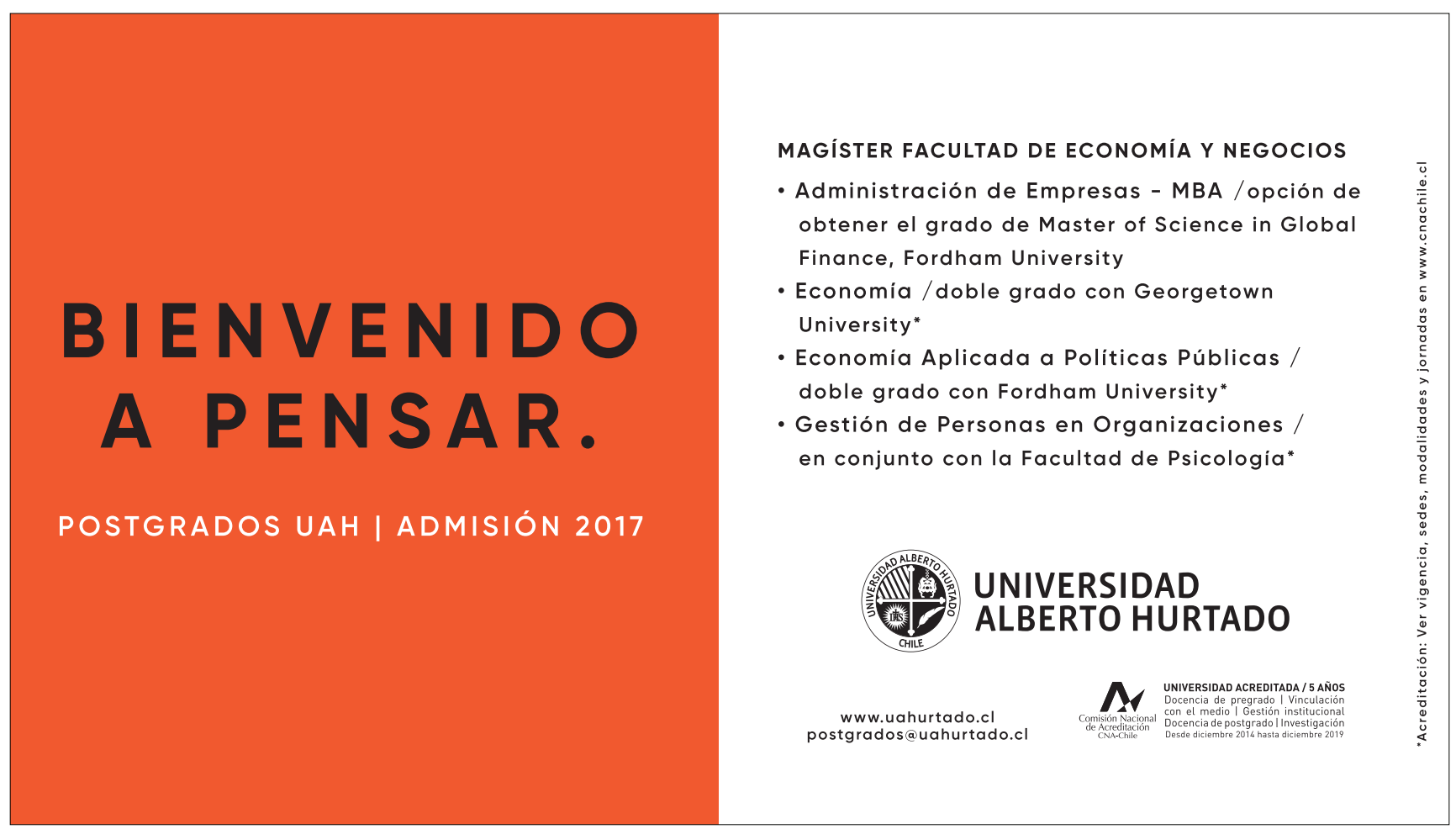

